\title{
Coalition Formation in Constant Sum Queueing Games
}

\author{
Shiksha Singhal ${ }^{1}$, Veeraruna Kavitha ${ }^{1}$ and Jayakrishnan Nair ${ }^{2}$ \\ $\mathrm{IEOR}^{1}, \mathrm{EE}^{2}$, Indian Institute of Technology Bombay, India
}

\begin{abstract}
We analyse a coalition formation game between strategic service providers of a congestible service. The key novelty of our formulation is that it is a constant sum game, i.e., the total payoff across all service providers (or coalitions of providers) is fixed, and dictated by the total size of the market. The game thus captures the tension between resource pooling (to benefit from the resulting statistical economies of scale) and competition between coalitions over market share. In a departure from the prior literature on resource pooling for congestible services, we show that the grand coalition is in general not stable, once we allow for competition over market share. Instead, the stable configurations are duopolies, where the dominant coalition exploits its economies of scale to corner a disproportionate market share. We analyse the stable duopolies that emerge from this interaction, and also study a dynamic variant of this game.
\end{abstract}

\section{INTRODUCTION}

Resource sharing is an efficient way of reducing congestion and uncertainty in service industries. It refers to an arrangement where service resources are pooled and used jointly by a group (a.k.a., coalition) of providers, instead of each provider operating alone using its own resources. Naturally, such a coalition would be sustainable only if the participating providers obtain higher payoffs than they would have obtained otherwise. The key driver of coalition formation in congestion prone service systems is the statistical economies of scale that emerge from the pooling of service resources-this allows the coalition to offer a better quality of service to its customers, and/or to attract more customers to its service.

Not surprisingly, there is a considerable literature that analyses resource pooling between independent providers of congestible services via a cooperative game theoretic approach. In these papers, each provider is modeled as a queueing system, with its own dedicated customer base, that generates service requests according to a certain arrival process. The payoff of each service provider is in turn determined by the quality of service it is able to provide to its (dedicated) customer base. In such a setting, the statistical economies of scale from resource pooling typically drives the service providers to pool all their servers together to form a grand coalition, which generates the greatest aggregate payoff across all coalitional arrangements. Naturally, the resulting aggregate payoff must be divided between the providers in a stable manner, i.e., in such a way that no subset of providers has an incentive to 'break away' from the grand coalition. Such stable payoff allocations have been demonstrated in a wide range of settings, including single/multiple server environments, and loss/queue-based environments (see [8], [9] and the references therein).

To summarize, the literature on coalition formation between providers of congestible services suggests that a stable grand coalition would emerge from the strategic interaction. However, a crucial aspect the preceding literature fails to capture is user churn. That is, customers can switch service providers, if offered superior service quality elsewhere. This aspect introduces competition between the service providers (or coalitions of service providers) over market share. To the best of our knowledge, the interplay between resource pooling among service providers (and the associated economies of scale) with the competition between them, in the context of congestible services, has not been explored in the literature. This paper seeks to fill this gap.

Specifically, we analyse a coalition formation game between a collection of service providers, each of which is modelled as an Erlang-B loss system. A key aspect of our model is that the total market size (captured via the aggregate arrival rate of customer requests) is fixed exogenously, making the game constant sum, i.e., the total payoff across all providers (or coalitions of providers) is fixed. This constant sum aspect, as we show, dramatically alters the outcome of the strategic interaction between providers. In particular, we show that (except in a very specific corner case), the grand coalition is not stable. Instead, the predominant stable configurations are duopolies, with the larger coalition exploiting economies of scale to corner a disproportionate portion of the market share. Our work also highlights several subtleties relating to different natural notions of stability in this context, the way the payoff of each coalition is divided between its members, and the degree of congestion in the system.

Our contributions are as follows.

1. We formally define a constant sum coalition formation game between strategic service providers of a congestible service. This model is the first, to the best of our knowledge, to capture the interplay between resource pooling and competition. Crucially, this is a partition form game, since the payoff of each coalition depends on not just the members of that coalition, but also on the coalitional arrangements outside the coalition.

2. We introduce three natural definitions of a stable configuration for this game, where a configuration specifies a partition of the set of providers (into coalitions), and also the allocation of the total payoff of each coalition among its members. The three notions of stability we consider differ with respect to the range of deviations or movements that are blocked (or disincentivised), and also the precision with which the coalitions that seek to 'break' from the prevailing 
configuration can estimate the benefit from doing so.

3. We analyse the class of stable configurations that emerge under each notion of stability. Interestingly, we are able to show that under configurations involving three or more coalitions are not stable under any notion of stability. Intuitively, this is because the economies of scale that incentivise certain mergers to take place between coalitions. Moreover, except for a corner case, we show that the grand coalition also cannot be part of a stable configuration. This means the dominant equilibria for this system are duopolies.

4. We further analyse the stable configurations that involve duopolies under each stability notion. Interestingly, the payoff allocations supported by stable configurations differ across the different stability notions. We also explore the impact of the overall congestion level on the stable duopolies, by analysing light and heavy traffic regimes.

5. Finally, we study a dynamic variant of the coalition formation game, and analyse the conditions for the (random) dynamics to 'settle' to a stable configuration in a finite number of moves.

\section{MOdel AND PRELIMINARIES}

In this section, we describe our system model for coalition formation between strategic service providers, characterize the behavior of the customer base in response to coalition formation between service providers, and introduce some background on the notion of a stable configuration.

\section{A. System model}

Consider a system with a set $\mathcal{N}=\{1, \cdots, n\}$ of independent service providers (a.k.a., agents), with provider $i$ having $N_{i}$ servers. Without loss of generality, we assume $N_{i} \geq N_{i+1}$ for $1 \leq i \leq n-1$. All servers are identical, and assumed to have a unit speed, without loss of generality. The providers serve a customer base that generates service requests as per a Poisson process of rate $\Lambda$. Jobs sizes (a.k.a., service requirements) are i.i.d., with $J$ denoting a generic job size, and $\mathbb{E}[J]=1 / \mu$.

Service providers are strategic, and can form coalitions with other service providers to enhance their rewards. Formally, such coalition formation between the service providers induces a partition $\mathcal{P}=\left\{C_{1}, C_{2}, \cdots, C_{k}\right\}$ of $\mathcal{N}$, where

$$
\cup_{i=1}^{k} C_{i}=\mathcal{N}, \quad C_{i} \cap C_{j}=\emptyset \forall i \neq j .
$$

We refer to such a partition with $k$ coalitions as a $k$-partition. (Naturally, the baseline scenario where each service provider operates independently corresponds to an $n$-partition.)

In response to a partition $\mathcal{P}$ induced by coalition formation between service providers, the arrival process of customer requests gets split across the $k$ coalitions in $\mathcal{P}$, with the arrival process seen by coalition $C$ being a Poisson process of rate $\lambda_{C}^{\mathcal{P}}$, where $\sum_{C \in \mathcal{P}} \lambda_{C}^{\mathcal{P}}=\Lambda$. (We characterize the split $\left(\lambda_{C}^{\mathcal{P}}, C \in \mathcal{P}\right)$ as a Wardrop equilibrium; details below.) Each coalition $C$ operates as an $M / M / N_{C} / N_{C}$ (Erlang-B) loss system, with $N_{C}=\sum_{j \in C} N_{j}$ parallel servers, and arrival rate $\lambda_{C}^{\mathcal{P}}$. This means jobs arriving into coalition $C$ that find a free server upon arrival begin service immediately, while those that arrive when all $N_{C}$ servers are busy get dropped (lost). Given the well known insensitivity property of the Erlang-B system, the steady state blocking probability associated with coalition $C$ (the long run fraction of jobs arriving into coalition $C$ that get dropped), denoted $B_{C}^{\mathcal{P}}$, is given by the Erlang-B formula ([11]):

$$
\begin{aligned}
& B_{C}^{\mathcal{P}}=B\left(N_{C}, a_{C}^{\mathcal{P}}\right), \text { where } a_{C}^{\mathcal{P}}:=\frac{\lambda_{C}^{\mathcal{P}}}{\mu}, \\
& B(N, a)=\frac{\frac{a^{N}}{N !}}{\sum_{j=0}^{N} \frac{a^{j}}{j !}} .
\end{aligned}
$$

\section{B. User behavior: Wardrop equilibrium}

Next, we define the behavior of the customer base in response to coalition formation across service providers, via the split $\left(\lambda_{C}^{\mathcal{P}}, C \in \mathcal{P}\right)$ of the aggregate arrival process of service requests across coalitions. This split is characterized as a Wardrop equilibrium ([1]).

In the context of our model, we define the WE split of the arrival process of service requests across coalitions, such that the steady state blocking probability associated with each coalition is equal. Note that since the blocking probability associated with an 'unused' coalition would be zero, it follows that all coalitions would see a strictly positive arrival rate. Thus, the WE (if it exists) is characterized by a vector of arrival rates $\left(\lambda_{C}^{\mathcal{P}}, C \in \mathcal{P}\right)$ satisfying,

$$
B_{C}^{\mathcal{P}}=B\left(N_{C}, \frac{\lambda_{C}^{\mathcal{P}}}{\mu}\right)=B^{*} \forall C \in \mathcal{P}, \quad \sum_{C \in \mathcal{P}} \lambda_{C}^{\mathcal{P}}=\Lambda,
$$

where $B^{*}$ is the common steady state blocking probability for each coalition. For any given partition $\mathcal{P}$, the following theorem establishes the existence and uniqueness of the WE, along with some useful properties.

Theorem 1: Given any partition $\mathcal{P}$ between the service providers, there is a unique Wardrop equilibrium $\left(\lambda_{C}^{\mathcal{P}}, C \in\right.$ $\mathcal{P}$ ), where $\lambda_{C}^{\mathcal{P}}>0$ for all $C \in \mathcal{P}$, that satisfies (2). Additionally, the following properties hold:

(i) For each $C \in \mathcal{P}, \lambda_{C}^{\mathcal{P}}$ is a strictly increasing function of the total arrival rate $\Lambda$.

(ii) If the partition $\mathcal{P}^{\prime}$ is formed by merging two coalitions $C_{i}$ and $C_{j}$ in partition $\mathcal{P}$ where $C_{i} \cup C_{j} \neq \mathcal{N}$ (with all other coalitions in $\mathcal{P}$ remaining intact),

$$
\lambda_{C_{i} \cup C_{j}}^{\mathcal{P}^{\prime}}>\lambda_{C_{i}}^{\mathcal{P}}+\lambda_{C_{j}}^{\mathcal{P}}
$$

(iii) If $\mathcal{P}=\left\{C_{1}, C_{2}\right\}$, with $N_{C_{1}}>N_{C_{2}}$, then

$$
\frac{\lambda_{C_{1}}^{\mathcal{P}}}{N_{C_{1}}}>\frac{\Lambda}{N}>\frac{\lambda_{C_{2}}^{\mathcal{P}}}{N_{C_{2}}} \text {, where } N=\sum_{i \in \mathcal{N}} N_{i}
$$

Proof: See Appendix B.

Aside from asserting the uniqueness and strict positivity of the Wardrop split, Theorem 1 also states that equilibrium arrival rate of each coalition is an increasing function of the aggregate arrival rate $\Lambda$; see Statement $(i)$. Additionally, Statement $(i i)$ demonstrates the statistical economies of scale due to a merger between coalitions: the merged entity is able to attract an arrival rate that exceeds the sum of the 
arrival rates seen by the two coalitions pre-merger. Finally, Statement $(\mathrm{iii})$ provides another illustration of statistical economies of scale for the special case of a 2-partition-the larger coalition enjoys a higher utilization per server than the smaller one.

\section{Coalition formation game: Preliminaries}

Having defined the behavior of the user base, we now provide some preliminary details on the coalition formation game between the service providers.

Recall, that each service provider is strategic, and only enters into a coalition if doing so is beneficial. Given a partition $\mathcal{P}$ that describes the coalitions formed by the service providers, we define the value or payoff of each coalition $C \in \mathcal{P}$ to be $\beta \lambda_{C}^{\mathcal{P}}$, where $\beta>0$. This is of course natural when the coalition derives a certain revenue per served job. The same model is also applicable if $\lambda_{C}^{\mathcal{P}}$ is interpreted as being proportional to the number of subscribers of coalition $C$, with each subscriber paying a recurring subscription fee. Without loss of generality, we set $\beta=1$.

The value $\lambda_{C}^{\mathcal{P}}$ of each coalition $C$ must further be apportioned between the members of the coalition. Denoting the payoff of agent $i$ by $\phi_{i}^{\mathcal{P}}$, we therefore have

$$
\sum_{i \in C} \phi_{i}^{\mathcal{P}}=\lambda_{C}^{\mathcal{P}} \quad \forall \quad C \in \mathcal{P} .
$$

Since the providers are selfish, they are ultimately interested only in their individual payoffs. Thus, the coalition formation between providers is driven by the desire of each provider to maximize its payoff, given the statistical economies of scale obtained via coalition, and also the constant sum nature of this game (the sum total of the payoffs of all providers equals $\Lambda$ ). Thus, the relevant fundamental questions are:

1) Which partitions can emerge as a result of the strategic interaction between providers, i.e., which partitions are part of stable configurations? Indeed, a precursor to this question is: How does one define a natural notion of stability?

2) It is apparent that the answer to the above question hinges on how the value of each coalition is divided between its members. Thus, the next question is: How is the value of each coalition apprortioned between its members in a stable configuration?

Our aim in this paper is to answer these questions; such problems can be studied using tools from cooperative game theory. Note in particular that the value of any coalition in our formulation depends on the operational arrangement of agents outside the coalition, i.e., on the entire partition. This makes the game we study a partition form game. In the remainder of this section, we introduce the notion of a partition (more precisely, a configuration) being blocked by a certain coalition. These ideas will be used when we define stable configurations in Section III.

Given a partition $\mathcal{P}=\left\{C_{1}, \cdots, C_{k}\right\}$, the set of payoff vectors consistent with $\mathcal{P}$ is defined as:

$\boldsymbol{\Phi}^{\mathcal{P}}:=\left\{\Phi=\left[\phi_{1}, \cdots, \phi_{n}\right] \in \mathbb{R}_{+}^{n}: \sum_{j \in C_{i}} \phi_{j}=\lambda_{C_{i}}^{\mathcal{P}} \forall 1 \leq i \leq k\right\}$
A configuration is defined as a tuple $(\mathcal{P}, \Phi)$, such that $\Phi \in$ $\boldsymbol{\Phi}^{\mathcal{P}}$. Note that a configuration specifies not just a partition of the agents into coalitions, but also specifies an allocation of payoffs within each coalition, that is consistent with the partition.

Blocking by a coalition: A configuration $(\mathcal{P}, \Phi)$ is blocked by a coalition $C \notin \mathcal{P}$ if, for any partition $\mathcal{P}^{\prime}$ containing $C$, there exists $\Phi^{\prime} \in \boldsymbol{\Phi}^{\mathcal{P}^{\prime}}$ such that

$$
\phi_{j}^{\prime}>\phi_{j} \forall j \in C \text {. }
$$

Basically, a new coalition can block an existing configuration, if each one of its members can derive strictly better payoff from this realignment. Equivalently, $(\mathcal{P}, \Phi)$ is blocked by coalition $C \notin \mathcal{P}$ if, for any partition $\mathcal{P}^{\prime}$ containing $C$, $\lambda_{C}^{\mathcal{P}^{\prime}}>\sum_{j \in C} \phi_{j}$. Note that the above equivalence hinges on the transferable utility assumption inherent in our cooperative game, by virtue of which (partial) utilities can be transferred across agents. Intuitively, a coalition $C \subset \mathcal{N}$ blocks configuration $(\mathcal{P}, \Phi)$, if the members of $C$ have an incentive to 'break' one or more coalitions of $\mathcal{P}$ to come together and form a new coalition. In particular, it is possible to allocate payoffs within the blocking coalition $C$ such that each member of $C$ achieves a strictly greater payoff, irrespective of any (potentially retaliatory) rearrangements among agents outside $C$. This is referred to in the literature as a pessimistic anticipation rule (see [4], [5] and Appendix A).

In Section III, we analyse stable configurations, which are defined as those configurations that cannot be blocked by a certain broad class of candidate blocking configurations. Specifically, we consider candidate blocking coalitions that are formed either via a merger of prevailing coalitions, or via a split of a single prevailing coalition. Also, note that blocking as defined above involves a revelation of the prevailing payoffs of the agents of the candidate coalition $\left\{\phi_{i}\right\}_{i \in C}$. In Section III, we also consider an alternative definition of blocking, where the 'prevailing worth' of the agents of the candidate blocking coalition is estimated imprecisely.

Finally, we note that game considered here can also be modelled as a characteristic form game; the details of this construction are available in Appendix A. Indeed, the notion of stable configurations in the present context is a partitionbased generalization of the classical notion of $\alpha$-core (see [2], [3]), when the characteristic function is defined using the pessimistic anticipation rule (details are in Appendix A, see (14)-(15).

\section{Stable CONFigurations}

In this section, we formally define three different notions of stable configurations, which differ based on the types of candidate blocking coalitions considered, as well as the precision with which the 'prevailing worth' of the members of the candidate coalition is estimated. For each of these notions of stability, we characterize the class of stable configurations. The main takeaway from our results is that the interplay between statistical economies of scale and the constant sum nature of this game results in configurations with three or more coalitions rendered unstable. In other 
words, stable configurations necessarily involve either a duopoly or a monopoly. Importantly, under all three notions of stability that we consider, stable configurations are only composed of such small (one/two) sized partitions; however, the payoff vector counterparts depend on the particular notion of stability under consideration.

We begin by defining the different notions of stability we consider.

\section{A. Defining stable configurations}

The first notion of stability we introduce simply restricts the set of candidate blocking configurations to mergers and splits of prevailing coalitions. Note that this is a natural restriction from a practical standpoint, since complex rearrangements between firms in a marketplace typically arise (over time) from a sequence of mergers and splits. We refer to this as restricted blocking (RB). Further when one assumes the precise knowledge of the worth of the blocking candidates, it leads to the RB-PA (Restricted BlockingPerfect Anticipation) rule. We begin with this rule.

RB-PA rule: Under this rule, a configuration $(\mathcal{P}, \Phi)$ is blocked by a coalition $Q$ that is formed either via a merger of coalitions in $\mathcal{P}$ (i.e., $Q=\cup_{C \in \mathcal{M}} C$ for $\mathcal{M} \subseteq \mathcal{P}$ ), or via the split of a single coalition in $\mathcal{P}$ (i.e., $Q \subset C$ for some $C \in \mathcal{P}$ ), if, for all partitions $\mathcal{P}^{\prime}$ containing $Q$, there exists $\Phi^{\prime} \in \boldsymbol{\Phi}^{\mathcal{P}^{\prime}}$ such that

$$
\phi_{i}^{\prime}>\phi_{i} \quad \forall \quad i \in Q
$$

Equivalently, $Q$ blocks the configuration $(\mathcal{P}, \Phi)$ if

$$
\underline{\lambda}_{Q}>\sum_{i \in Q} \phi_{i}, \text { where } \underline{\lambda}_{Q}:=\min _{\mathcal{P}^{\prime}: Q \in \mathcal{P}^{\prime}} \lambda_{Q}^{\mathcal{P}^{\prime}} \text {. }
$$

A configuration $(\mathcal{P}, \Phi)$ is stable under the RB-PA rule if it is not blocked by any merger or split. Note that under the RB-PA rule, members of a candidate blocking coalition are pessimistic in their anticipation of the value of the new coalition, in that they consider 'worst case' rearrangements among outside agents. Moreover, it is possible to allocate the payoff of $Q$ among its members such that each member is (strictly) better off, as discussed in previous section.

The next notion we consider uses the same restriction on the set of candidate blocking configurations, but uses an imprecise estimate of the prevailing worth of the members of the candidate blocking configurations in the case of a split, resulting in an imprecise anticipation of the benefit from the split. We refer to this as the RB-IA (Restricted BlockingImperfect Anticipation) rule.

RB-IA rule: Under this rule, a configuration $(\mathcal{P}, \Phi)$ is blocked by a coalition $Q$ that is formed by splitting a coalition $C \in \mathcal{P}$ if:

$$
\begin{aligned}
& \underline{\lambda}_{Q}:=\min _{\mathcal{P}^{\prime}: Q \in \mathcal{P}^{\prime}} \lambda_{Q}^{\mathcal{P}^{\prime}}>\frac{N_{Q}}{N_{C}} \lambda_{C}^{\mathcal{P}}, \\
& \lambda_{Q}^{\hat{\mathcal{P}}}>\sum_{i \in Q} \phi_{i}, \text { where } \hat{\mathcal{P}}=(\mathcal{P} \backslash\{C\}) \cup\{Q, C \backslash Q\} .
\end{aligned}
$$

Observe here that the right hand side expression in (5) is an imprecise estimate of the worth of the breaking away split, while that in the second equation is the precise value (to be revealed in later part of the negotiations). On the other hand, under the RB-IA rule, a configuration $(\mathcal{P}, \Phi)$ is blocked by a coalition $Q$ that is formed by a merger of coalitions in $\mathcal{P}$ if

$$
\underline{\lambda}_{Q}>\sum_{C \subset Q} \lambda_{C}^{\mathcal{P}}, \text { and } \lambda_{Q}^{\hat{\mathcal{P}}}>\sum_{i \in Q} \phi_{i},
$$

where $\hat{\mathcal{P}}$ is the new partition after the merger. Observe here that $\sum_{i \in Q} \phi_{i}=\sum_{C \subset Q} \lambda_{C}^{\mathcal{P}}$ and hence the second condition is immediately satisfied for merger, because $\underline{\lambda}_{Q} \leq \lambda_{Q}^{\hat{\mathcal{P}}}$. Finally, a configuration is stable under the RB-IA rule if it is not blocked by any merger or split.

Note that RB-PA and RB-IA differ only in the condition for blocking due to a split. This is natural, since the net worth of coalitions $\left\{\lambda_{C}^{\mathcal{P}}\right\}_{C \in \mathcal{P}}$ is often common knowledge, whereas the internal payoff allocation within a coalition can often be confidential. Let us therefore interpret the condition for blocking due to a split under RB-IA. Condition (5) can be interpreted as a first stage check on the feasibility of the split, by (imperfectly) estimating the total prevailing worth of the members of $Q$ as proportional to their contribution to the service capacity within $C$. On the other hand, the condition (6) can be interpreted as the final stage check on split feasibility, that ensures that it is possible to allocate the payoff of $Q$ among its members such that each member is (strictly) better off from the split.

Finally, we consider the stability notion resulting from the most general model for blocking. Here, we allow blocking by an arbitrary coalition (which also includes merger of partial splits), with a precise estimation of the prevailing worth of the members of the blocking coalition. We refer to this as the GB-PA (General Blocking-Perfect Anticipation) rule.

GB-PA rule: Under this rule, a configuration $(\mathcal{P}, \Phi)$ is blocked by any coalition $Q \notin \mathcal{P}$ if (4) holds. A configuration is stable under the GB-PA rule if it is not blocked by any coalition.

Clearly, the set of stable configurations under the GB-PA rule is a subset of the set of stable configurations under the RB-PA rule.

Having defined our notions of stability, we now consider each notion separately, and characterize the resulting stable configurations. We begin with RB-IA, which (it turns out), admits the broadest class of stable configurations.

\section{B. Stable configurations under RB-IA}

Our first main result is that all configurations involving partitions of size three or more are unstable. In other words, only monopolies or duopolies can be stable.

Theorem 2: Under the RB-IA rule, any configuration $(\mathcal{P}, \Phi)$ with $|\mathcal{P}| \geq 3$ is not stable.

Proof: See Appendix C.

Intuitively, partitions of size three or more are unstable because of the statistical economies of scale resulting from a merger (see Statement (ii) of Theorem 1). Specifically, if $|\mathcal{P}|=k \geq 3$, it can be shown that any merger between $k-1$ coalitions in $\mathcal{P}$ would block the configuration $(\mathcal{P}, \Phi)$.

Having ruled out the possibility of stable configurations with three or more coalitions, we now explore the two 
remaining possibilities: stable configurations involving the grand coalition, and those involving 2-partitions.

Grand Coalition: Defining $\mathcal{P}_{G}:=\mathcal{N}$ as the grand coalition, it is clear that any configuration of the form $\left(\mathcal{P}_{G}, \Phi\right)$ can only be blocked by a split. We now show that unless a single agent owns more than half the total service capacity of the system, such a block is always possible. In other words, any configuration involving the grand coalition is unstable unless there is a single 'dominant' agent. On the other hand, if there is a single agent who owns more than half the service capacity, we show that there exists stable configurations of the form $\left(\mathcal{P}_{G}, \Phi\right)$, i.e., the grand coalition can be stable in the presence of single dominant agent.

Theorem 3: Under the RB-IA rule:

i) If $N_{1} \leq \sum_{i \in \mathcal{N} ; i \neq 1} N_{i}$, there exists no payoff vector $\Phi$ consistent with $\mathcal{P}_{G}$, such that $\left(\mathcal{P}_{G}, \Phi\right)$ is stable.

ii) If $N_{1}>\sum_{i \in \mathcal{N} ; i \neq 1} N_{i}$, there exists atleast one payoff vector $\Phi$ consistent with $\mathcal{P}_{G}$, such that $\left(\mathcal{P}_{G}, \Phi\right)$ is stable.

Proof: See Appendix C.

Two-partitions: We finally turn to configurations involving 2-partitions. Two-partitions can, without loss of generality, be represented as $\mathcal{P}=\left\{C_{1}, C_{2}\right\}$, with $N_{C_{1}} \geq N_{C_{2}}$. We now show that under the RB-IA rule, the stability/instability of a configuration $(\mathcal{P}, \Phi)$ depends majorly on the value of $k:=$ $N_{C_{1}}$. Interestingly, the stability/instability of a configuration is generally not influenced by the associated payoff vector $\Phi$ under the RB-IA rule. (This is not true under RB-PA or GBPA rules.)

Formally, let $\lambda_{k}:=\lambda_{C_{1}}^{\mathcal{P}}$. Note that by Theorem $1, \lambda_{k}$ is the unique zero of the following function of $\lambda$ (see (2)):

$$
h(\lambda):=\frac{\lambda^{k}}{k !} \sum_{j=0}^{N-k} \frac{(\Lambda-\lambda)^{j}}{j !}-\frac{(\Lambda-\lambda)^{N-k}}{(N-k) !} \sum_{j=0}^{k} \frac{\lambda^{j}}{j !} .
$$

Next, define $\Psi(k ; \Lambda):=\lambda_{k} / k$ as the utilization per server of the larger coalition. Finally, define

$$
k^{*}(\Lambda):=\underset{k: k=N_{C_{1}}}{\arg \max } \Psi(k ; \Lambda) .
$$

Note that $k^{*}(\Lambda)$ is the set of values of $k$ that maximizes the per-server utilization of the larger coalition.

Let $\mathbb{C}^{*}:=\left\{C \subset \mathcal{N}: N_{C} \in k^{*}(\Lambda)\right\}$ be the set of coalitions $C$, that can derive maximum per-server utilization, irrespective of the operational arrangement of the other agents. In the following lemma, we provide a sufficient condition for a class of configurations to be stable. We adopt the following convention: A partition $\mathcal{P}$ is stable if all configurations involving it are stable, i.e., configuration $(\mathcal{P}, \Phi)$ is stable for any $\Phi \in \boldsymbol{\Phi}^{\mathcal{P}}$.

Lemma 1: Consider the RB-IA rule. A 2-partition $\mathcal{P}=$ $\left\{C_{1}, C_{2}\right\}$ is stable if there exists no coalition $S \subset C_{i}$ for $i=$ $\{1,2\}$ such that: $\frac{\underline{\lambda}_{S}}{N_{S}}>\frac{\lambda_{C_{i}}}{N_{C_{i}}}=\frac{\lambda_{C_{i}}^{\mathcal{P}}}{N_{C_{i}}}$.

The proof of the lemma follows directly from the definition of stability. A consequence of this lemma is the following.

Theorem 4: Consider the RB-IA rule. Any 2-partition $\mathcal{P}=\left\{C_{1}, C_{2}\right\}$ with one of the coalitions from $\mathbb{C}^{*}$ is a stable partition. Additionally, any partition $\mathcal{P}=\left\{C_{1}, C_{2}\right\}$ satisfying $N_{C_{1}}=N_{C_{2}}=N / 2$ is stable.

Proof: See Appendix C.

Note that under the RB-IA rule, we have identified a class of stable partitions, i.e., these partitions are stable for any consistent payoff vector. In Section V], we provide a complete characterization of the class of stable partitions under RB-IA, in the heavy and light traffic regimes.

\section{Stable configurations under RB-PA}

Next, we consider stable configurations under the RB-PA rule, which presents interesting contrasts to the RB-IA rule. Under this rule, we show that only coalitions involving 2partitions can be stable, i.e., configurations involving the grand coalition, or involving $k$-partitions with $k \geq 3$ are always unstable. Moreover, the stability/instability of configurations involving 2-partitions depends on the associated payoff vector.

To demonstrate this, we define a special proportional payoff vector, where the value of each coalition is divided between its members in proportion to the number of servers they bring to the coalition. Formally, the proportional payoff vector $\Phi_{p}^{\mathcal{P}}$ associated with a partition $\mathcal{P}$ is defined by:

$$
\phi_{p, i}^{\mathcal{P}}=\frac{N_{i}}{\sum_{j \in C} N_{j}} \lambda_{C}^{\mathcal{P}} \text { for any } i \in C \in \mathcal{P} .
$$

Our results for the RB-PA rule are summarized as follows.

Theorem 5: Under the RB-PA rule:

i) No configuration involving the grand coalition is stable. ii) No configurations involving $k$-partitions, where $k \geq 3$ are stable.

iii) For any 2-partition $\mathcal{P}=\left\{C_{1}, C_{2}\right\}$, where one of the coalitions lies in $\mathbb{C}^{*},\left(\mathcal{P}, \Phi_{p}^{\mathcal{P}}\right)$ is stable.

iv) For any 2-partition $\mathcal{P}=\left\{C_{1}, C_{2}\right\}$, where $N_{C_{1}}=N_{C_{2}}=$ $N / 2,\left(\mathcal{P}, \Phi_{p}^{\mathcal{P}}\right)$ is stable.

v) More generally, consider any 2-partition $\mathcal{P}=\left\{C_{1}, C_{2}\right\}$, that is stable under $R B-I A$ rule. Then $\left(\mathcal{P}, \Phi_{p}^{\mathcal{P}}\right)$ is stable under $R B-P A$ rule. Further there exists a neighbourhood $\mathcal{B}_{p}^{\mathcal{P}}$ of the payoff vector $\Phi_{p}^{\mathcal{P}}$ such that $(\mathcal{P}, \Phi)$ is stable for all $\Phi \in \mathcal{B}_{p}^{\mathcal{P}}$. Proof: See Appendix C.

Theorem 5 conveys that partitions that are stable under the RB-IA rule (irrespective of the associated payoff vector), are also part of stable configurations under RB-PA, but under a restricted class of payoff vectors. Specifically, the payoff vectors we identify are 'close' to proportional allocations. Whether there are other natural payoff structures that also induce stability under RB-PA, is an interesting question for future work.

\section{Stable configurations under GB-PA}

Finally, we consider the GB-PA rule, which allows for a configuration to be blocked by any arbitrary coalition, formed via (possibly) simultaneous partial splits and partial mergers. This is also the case in the general definition of blocking by a coalition as given in Section II as well as in [5] and Appendix A. We briefly discuss this case here; a complete 
characterization of stability under GB-PA will be pursued as future work.

It is immediate that since pure mergers or pure splits are included within the class of candidate blocking coalitions under GB-PA, any configuration that is not stable under RB$P A$ is also not stable under GB-PA. From Theorem 5, it therefore follows that the grand coalition, and $k$-partitions with $k \geq 3$, can never be part of a stable configuration under GB-PA.

Now, consider the following stable configuration (under the RB-PA rule) from the same theorem, $\left(\mathcal{P}, \Phi_{p}^{\mathcal{P}}\right)$ with $\mathcal{P}=\{C, \mathcal{N} \backslash C\}$, and $C \in \mathbb{C}^{*}$. If there exists $S \subset C$ such that $\left.C^{\prime}:=S \cup(\mathcal{N} \backslash C)\right\} \in \mathbb{C}^{*}$, then $C^{\prime}$ would block the configuration $\left(\mathcal{P}, \Phi_{p}^{\mathcal{P}}\right)$, since

$$
\begin{aligned}
\sum_{i \in C^{\prime}} \phi_{p, i}^{\mathcal{P}} & =\sum_{i \in S} \phi_{p, i}^{\mathcal{P}}+\sum_{i \in \mathcal{N} \backslash C} \phi_{p, i}^{\mathcal{P}}=N_{S} \frac{\lambda_{C}^{\mathcal{P}}}{N_{C}}+N_{\mathcal{N} \backslash C} \frac{\lambda_{\mathcal{N} \backslash C}^{\mathcal{P}}}{N_{\mathcal{N} \backslash C}} \\
& <N_{S} \frac{\lambda_{C}^{\mathcal{P}}}{N_{C}}+N_{\mathcal{N} \backslash C} \frac{\lambda_{C}^{\mathcal{P}}}{N_{C}}=\left(N_{C^{\prime}}\right) \frac{\lambda_{C^{\prime}}^{\mathcal{P}^{\prime}}}{N_{C^{\prime}}}=\underline{\lambda}_{C^{\prime}}
\end{aligned}
$$

where $\mathcal{P}^{\prime}=\left\{C^{\prime}, \mathcal{N} \backslash C^{\prime}\right\}$. This suggests that stability under GB-PA is more fragile as compared to (the arguably more practical) RB-PA rule. It would thus be interesting to investigate the following questions in the future: a) Do there exist stable configurations under GB-PA (i.e., under the broadest class of blocking coalitions)? b) In a dynamic game environment (of the kind considered in Section IV), would GB-PA induce a limit cycle between certain equivalent configurations?

\section{DyNAMiC COALITION FORMATION GAME}

In this section we consider a dynamic version of the game discussed in the previous sections. We begin with a queueing system and agents operating in some configuration. The agents are constantly on the lookout for greener pastures, and would stop their quest only if they are satisfied with the existing configuration.

Agents may consider joining existing collaborations or may consider splitting from some of them. The (new) payoffs derived by the agents after the new collaborations (if any), depend upon the previous payoffs and the value of the new operational arrangement/coalition. Depending upon the new payoffs, some of the agents might again consider another movement. On the other hand, the system might settle, if all the agents are satisfied with the configuration. We study this aspect by considering a sequence of dynamic coalition formations.

Dynamics: The system starts with some operational arrangement given by $\mathcal{P}_{0}$ and with a payoff vector $\Phi^{0}=$ $\left[\phi_{1}^{0}, \cdots, \phi_{n}^{0}\right]$. If the configuration $\left(\mathcal{P}_{0}, \Phi^{0}\right)$ is stable as defined in previous sections, it is not beneficial for any member to consider any (coalitional) deviation and hence the system does not undergo any change. If that is not the case, some members of the partition merge/split.

There could be more than one movement (merger/split) that may be successful, under both the assessment rules (RB-PA and RB-IA). We assume that any such blocking coalition $Q$ is equally likely to form, causing the system to evolve to a new partition, say $\mathcal{P}_{1}$. In case of the RB-IA rule, any new payoff vector $\Phi^{1}$ that satisfies $\phi_{i}^{1}>\phi_{i}^{0}$ for all $i \in Q$ would suffice. We discuss the RB-PA rule towards the end of this section.

The system stops if the new configuration $\left(\mathcal{P}_{1}, \Phi^{1}\right)$ is stable. If not, it switches to yet another configuration $\left(\mathcal{P}_{2}, \Phi^{2}\right)$ randomly (and equally likely among all possible movements) in a similar way. This evolution continues until stopped by a stable configuration. Our aim is to understand if such a limit stable configuration exists.

By the results of the previous section we have stable configurations only with 2-partitions or grand coalition and we immediately have the following result under the following assumption:

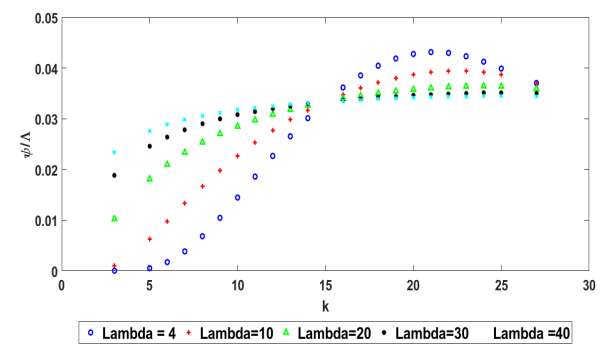

Fig. 1. $\Psi(k ; \Lambda) / \Lambda \mathrm{v} / \mathrm{s} k$, with $\left[N_{i}\right]=[9,7,6,5,3]$.

A.1) If $C$ is any coalition that does not contain any element of $\mathbb{C}^{*}$, i.e., if $C \cap C^{*} \neq C^{*}$ for all $C^{*} \in \mathbb{C}^{*}$, then we have the following:

$$
\frac{\underline{\lambda}_{S}}{N_{S}}<\frac{\underline{\lambda}_{C}}{N_{C}} \text { for all strict subtsets, } S \subset C .
$$

Basically this assumption ensures that any 2-partition that is not stable, necessarily contains a coalition that is a strict superset of an element from $\mathbb{C}^{*}$. From simulations, we have seen that this assumption is satisfied by our queuing system for all the cases that we considered (for example, see Figure 1) and further by Theorem 7 and Lemma 3 can be shown to hold under heavy and light traffic conditions. Under this assumption, we can show that the dynamics stops after finite number of movements.

Theorem 6: [Convergence] Assume A.1. Then the random dynamics under RB-IA rule converges to one of the stable configurations under RB-IA rule in finite number of steps, with probability one.

Proof: We first show that starting from any $k$-partition $\mathcal{P}$ with $k>2$, the dynamics hits a 2-partition with probability one: i) from any such $\mathcal{P}$, there exists at least one direct path to a 2-partition with probability strictly greater than zero, as given in the proof of Theorem 2, ii) thus there exists a non-zero uniform lower bound $\underline{p}>0$ on the probability of hitting a 2-partition, irrespective of the starting $k$-partition, because of finitely many such partitions; and iii) thus by independence, the dynamics hits a 2-partition with probability one in finite number of steps (uniformly upper bounded by a geometric random variable with parameter $\underline{p}$ ).

Similarly starting from the grand coalition the system either evolves to a 2-partition or stops. 
If the dynamics hits one of the stable partitions (among 2partitions), we are done. If not, by A.1, the 2-partition (say $\mathcal{P}=\{C, \mathcal{N} \backslash C\}$ ) is such that (without loss of generality) $N_{C}>k^{*}$ and $C$ contains a $C^{*} \in \mathcal{C}^{*}$. The movement from $\mathcal{P}$ to $\mathcal{P}_{1}:=\left\{C^{*}, C \backslash C^{*}, \mathcal{N} \backslash C\right\}$ is possible by (5) because clearly by definition of $k^{*}$ and $C^{*}$

$$
\frac{\underline{\lambda}_{C^{*}}}{k^{*}}>\frac{\lambda_{C}}{N_{C}}=\frac{\lambda_{C}^{\mathcal{P}}}{N_{C}} \text { which implies } \underline{\lambda}_{C^{*}}>\lambda_{C}^{\mathcal{P}} \frac{k^{*}}{N_{C}} .
$$

From $\mathcal{P}_{1}$ merger of $C \backslash C^{*}$ and $\mathcal{N} \backslash C$ to $\mathcal{P}_{2}:=\left\{C^{*}, \mathcal{N} \backslash C^{*}\right\}$ is possible by (7), as clearly

$$
\underline{\lambda}_{\mathcal{N} \backslash C^{*}}>\lambda_{C \backslash C^{*}}^{\mathcal{P}_{1}}+\lambda_{\mathcal{N} \backslash C^{*}}^{\mathcal{P}_{1}},
$$

as in the proof of Theorem 2. The succession of these two events occur with probability that can be lower bounded by a strictly positive number $p^{\prime}$, uniformly across all such starting 2-partitions. As in the previous paragraph, any upward movement will return to a 2-partition with probability one and in each of these returns there is uniform lower bound $\underline{p}^{\prime}$ on the probability of return to the stable 2-partition with a $C^{*}$. Hence the theorem.

The above theorem proves that the random dynamics under RB-IA rule is stopped in finite number of steps with probability one, and the limit is a stable partition. However under this imprecise anticipation rule, it is important to observe that the payoff vector at the stopped configuration can be arbitrarily skewed (as also indicated in Theorem 4).

\section{Dynamics under RB-PA rule}

Under RB-PA rule, the random dynamics behaves exactly similar to RB-IA rule (as described in the proof), however it may not stop even after touching a stable 2-partition, stable under RB-IA. As seen from Theorem 5 for RB-PA rule, the payoff vector is equally important in the definition of stable configuration.

This shows the importance of appropriate reallocation of individual shares after the new move towards the stability of the new system; it is not sufficient to only ensure all members of the new coalition derive positive increments, rather we will require that the new allocation matches the payoff vector in the corresponding stable configuration. As seen from Theorem 5 , one of the payoff vectors that provides stable configurations is the proportional payoff vector given by equation (9). Thus one probably has to design reallocation policies that converge towards the proportional payoff vectors for the dynamics under the RB-PA rule to stop.

Alternatively there might be other payoff vectors which would also form a part of the stable configurations and they could be the ones at limit. We would study this aspect in the future, but for now we could say that one can't have partitions of size greater than 2 or the grand coalition (when none of the agents dominate) to be a part of the limit (stable) configuration (if one exists), in view of Theorems 2 and 3 . We can also say that the dynamics stops if it hits upon a configuration with stable 2-partition and the corresponding proportional payoff vector (9).

\section{Dynamics under GB-PA rule}

We only have preliminary results for this rule. As mentioned before, all the configurations that we discussed before are not stable. It is not difficult to show that the dynamics does not stop even if it starts with or hits a stable configuration under RB-PA rule identified in Theorem 5 It is interesting to observe that the dynamics toggles between stable configurations of RB-PA rule, even when it starts with one of them.

\section{Stable 2-PARtitions}

We now obtain the stable partitions of Theorems 4 (RB-IA) and 5 (RB-PA). We achieve this by considering heavy and light traffic regimes. Recall any 2-partition $\mathcal{P}=$ $\left\{C_{1}, C_{2}\right\}$ can be identified uniquely by $k:=N_{C_{1}}, \lambda_{k}:=$ $\underline{\lambda}_{C_{1}}$, when one considers optimizing $\Psi(k ; \Lambda)=\lambda_{k} / k$. We first begin with analysis of $k^{*}$ defined in (8).

\section{A. Heavy Traffic}

Our aim in this section is to derive the analysis using some appropriate approximations, and then prove that the derived results are valid for for all arrival rates with $\Lambda>\bar{\Lambda}$, where $\bar{\Lambda}$ is a big enough value.

The WE can also be obtained by equating the reciprocal of blocking probabilities (1) and the first order approximation suggests that approximate WE can be obtained by solving the following equation written in terms of $\lambda_{1}$ and $\lambda_{2}:=\Lambda-\lambda_{1}$, $1+\frac{k}{\lambda_{1}}=1+\frac{N-k}{\lambda_{2}}$ and then the solution $\lambda_{1}^{*}=\frac{k}{N} \Lambda$.

Thus with this approximation the share of any agent $i \in C_{1}$ under proportional payoffs 9 equals $N_{i} / k * k / N \Lambda=$ $N_{i} / N \Lambda$, irrespective of $k$. Thus this approximation is not sufficient and we now consider the second order approximation under which we require zeros of the following:

$1+\frac{k}{\lambda_{1}}+\frac{k(k-1)}{\lambda_{1}^{2}}=1+\frac{N-k}{\lambda_{2}}+\frac{(N-k)(N-k-1)}{\lambda_{2}^{2}}$,

which after some simple calculations leads to the following fixed point equation (of $\lambda_{1} \in[0, \Lambda]$ and $\lambda_{2}:=\Lambda-\lambda_{1}$ ):

$$
\frac{\lambda_{1}}{k}=\frac{\lambda_{2}}{N-k}\left(\frac{1+\frac{k}{\lambda_{1}}-\frac{1}{\lambda_{1}}}{1+\frac{N-k}{\lambda_{2}}-\frac{1}{\lambda_{2}}}\right) .
$$

Let $\psi(k):=\hat{\lambda}_{k} / k$ where $\hat{\lambda}_{k}$ is the fixed point of the above function and observe that $\Psi(\cdot)$ represents similar function, but considering exact blocking probability (1). For further analysis, we relax $k$ to be a real value between $(N / 2, N)$. We immediately have the following result:

Lemma 2: i) There exists a $\bar{\Lambda}$ such that, the function $\psi$ is increasing with $k$, for any $\Lambda \geq \bar{\Lambda}$.

ii) For any $\Lambda \geq \bar{\Lambda}$, under second order approximation, the unique maximizer in (8) is given by $k^{*}=\sum_{i=1}^{n-1} N_{i}$.

iii) For all such $\Lambda$, the partitions $\mathcal{P}=\{C, \mathcal{N} \backslash C\}$, with $N / 2 \leq N_{C} \leq k^{*}$ are the only 2-partitions that are stable, under second order approximation.

Proof: See Appendix C. 
Accuracy of the approximation We now prove that the above result is also true without approximation using maximum theorem [10]. We will show that both the fixed points converge towards each other and that there exists a $\bar{\Lambda}$ such that, stable partition considering true blocking probability equals that derived with second order approximation.

Consider $y \in[\epsilon, 1-\epsilon]$ for some small $\epsilon>0$ and define the following function:

$$
g(y, \theta)= \begin{cases}\sqrt{\frac{1}{\theta}}\left(\sum_{j=0}^{k-1} \frac{(y / \theta)^{j-k}}{j !} k !\right. & \\ \left.-\sum_{j=0}^{(N-k)-1} \frac{[(1-y) / \theta]^{j-(N-k)}}{j !}(N-k) !\right)^{2}, & \text { if } \theta>0 \\ \left(y^{-1} k-(1-y)^{-1}(N-k)\right)^{2} & \text { if } \theta=0 .\end{cases}
$$

Observe from (1) that $y=\frac{\lambda_{1}}{\Lambda}$ (the normalized WE) is the unique zero of the function $g($.$) when \Lambda=1 / \theta$, uniqueness given by Theorem 1, and the solution by first order approximation is zero of $g$ when $\theta=0$. It is clear that $g$ is a jointly continuous mapping ${ }^{1}$ over $[\epsilon, 1-\epsilon] \times[0, B]$ (for any $B<\infty$ ). Define,

$g^{*}(\theta) \triangleq \max _{y \in[\epsilon, 1-\epsilon]} g(y, \theta)$ and $y^{*}(\theta) \triangleq \arg \max _{y \in[\epsilon, 1-\epsilon]} g(y, \theta)$.

Then, by Maximum Theorem, $y^{*}(1 / \Lambda) \rightarrow y^{*}(0)$ as $\Lambda \rightarrow \infty$. In other words we have:

$$
\frac{\lambda_{1}(\Lambda)}{\Lambda} \rightarrow \frac{k}{N} \text {, or equivalently, } \frac{1}{\Lambda}\left|\frac{\lambda_{1}^{*}}{k}-\frac{\Lambda}{N}\right| \rightarrow 0
$$

Using exactly similar logic, one can show that the WE using second order approximation also converges towards that of the first order approximation, and hence that the differences between the WE obtained using second order approximation and that obtained using true blocking probability (1) converge towards each other. Using this we prove:

Theorem 7: There exists a $\bar{\Lambda}$ such that, only $k^{*}:=$ $\sum_{i=1}^{n-1} N_{i}$ optimizes (8) for our queueing system with any $\Lambda \geq \bar{\Lambda}$. Further the only partitions that are part of a stable configuration (under $R B-P A / R B-I A$ ) are the 2-partitions $\mathcal{P}=$ $\{C, \mathcal{N} \backslash C\}$, with $N / 2 \leq N_{C} \leq k^{*}$.

Proof: By the above arguments for any 2-partition $\mathcal{P}=$ $\left(C_{1}, C_{2}\right)$ with $N_{C_{1}}=k$, the the WE obtained using second order approximation (10), represented by $\hat{\lambda}_{k}$, and the one obtained using the exact blocking probability (1) converge towards each other for all $k$ as $\Lambda \rightarrow \infty$. Consider $\bar{\Lambda}$ further large in Lemma 2 such that (possible by finiteness)

$\frac{1}{\Lambda}\left|\hat{\lambda}_{k}(\Lambda)-\lambda_{k}(\Lambda)\right|<\delta$ for all possible $k$, and for $\Lambda \geq \bar{\Lambda}$,

where $\delta>0$ is sufficiently small so that the conclusions of the theorem follow from that in Lemma 2 (possible by finitely many values of $k$ ), after observing that monotonicity of $\Psi$ with respect to $k$ for any given $\Lambda$ is equivalent to monotonicity of $\Psi / \Lambda$ with respect to $k$.

\footnotetext{
${ }^{1}$ If $\epsilon$ is such that the true WE does not fall in interval $[\epsilon, 1-\epsilon]$, then we would have some other points as the minimizers of $g($.$) (by continuity and$ compactness), but eventually (with large enough $\Lambda$ ) we will have unique zero of $g($.$) which is derived from unique WE of the original problem.$
}

\section{B. Light Traffic}

We now consider light traffic regimes, and derive the following result using similar logic as before:

Lemma 3: There exists a $\underline{\Lambda}>0$, such that for all $\lambda<$ $\underline{\Lambda}$, we have: i) $\underline{k}^{*}:=\min \left\{N_{C}: N_{C}>N / 2, C \subset \mathcal{N}\right\}$ optimizes (8) for our queueing system; and ii) Further the only partitions that are part of a stable configuration (under $R B-P A / R B-I A)$ are the 2-partitions $\mathcal{P}=\{C, \mathcal{N} \backslash C\}$, with $N / 2 \leq N_{C} \leq k^{*}$.

Proof: See Appendix C.

Thus in both (light as well as the heavy) the traffic regimes, stable partitions are the 2-partitions with $N / 2 \leq k \leq k^{*}$. Further observe that assumption A.1 is satisfied for both these regimes. Hence the random dynamics under RB-IA, in either case, converges and stops at one of such 2-partitions.

\section{NUMERICAL COMPUTATIONS}

In this section, we simulate two systems with 5 agents. The agents in first and second system have 9, 7, 6, 5, 3 and 10, 7, 6, 5, 4 servers respectively. From left sub-figure of Figure 2. we have that $k^{*}$ is a monotonically increasing function of $\Lambda$ in both cases. The corresponding right sub-figure shows blocking probability as a function of $\Lambda$ in both cases. One
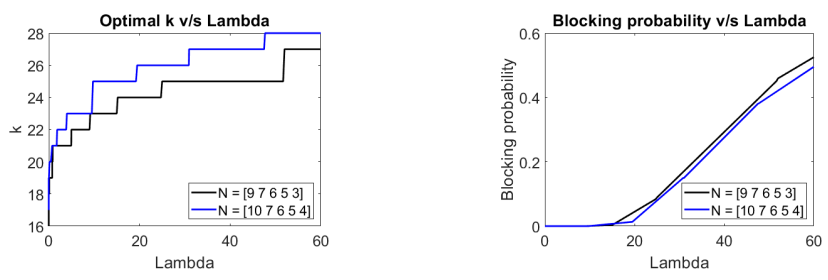

Fig. 2. Optimal $k^{*}$ v/s $\Lambda$ (left) and $B$ v/s $\Lambda$ (right)

may observe that the end points of the left sub-figure, i.e., under heavy and low traffic matches with the ones derived in Theorem 7 and Lemma 3.

\section{CONCLUSIONS}

We consider a queueing system with several strategic service providers with different server capabilities. They are on lookout for collaboration opportunities that improve their individual payoffs. The customer base responds to any operational arrangement formed by such collaborations, the customer arrivals are split across various operational units according to the well known Wardrop equilibrium that equalizes the steady state blocking probability of all the units. Any operational configuration is challenged by new coalition formed by mergers or splits, and the former is dissolved if the new coalition finds benefit. A configuration is stable if there is no coalition to challenge it. We defined three notions of stability and our major findings are: a) configurations with more than two coalitions are never stable; b) grand coalition can be stable depending upon the payoff allocations and the notion of stability, only if there exists a single dominant player with more than half the server capacity of the system; and c) some configurations with two coalitions are stable, depending upon the notion of stability and the 
payoff allocations. We also consider an initial model with dynamic coalition formations and showed the convergence of the same under one notion of stability. This work just opened an array of questions that need exploration.

\section{REFERENCES}

[1] Correa, José R and Stier-Moses, Nicolás E, Wardrop equilibria Wiley encyclopedia of operations research and management science, 2010.

[2] Aumann, Robert J, The core of a cooperative game without side payments. Transactions of the American Mathematical Society, vol. 98, no. 3, pp. 539-552, 1961.

[3] Martins-da-Rocha, Victor Filipe and Yannelis, Nicholas C, Nonemptiness of the alpha-core. Fundação Getulio Vargas. Escola de Pósgraduação em Economia, 2011.

[4] Bloch, Francis and Van den Nouweland, Anne, Expectation formation rules and the core of partition function games, Games and Economic Behavior, vol. 88, pp. 339-353, 2014.

[5] Shiksha Singhal and Veeraruna Kavitha, Coalition Formation Resource Sharing Games in Networks Accepted in Performance Evaluation, 2021.

[6] Hajduková, Jana, Coalition formation games: A survey International Game Theory Review, vol. 8, no. 04, pp. 613-641, 2006.

[7] Saad, Walid and Han, Zhu and Debbah, Mérouane and Hjorungnes, Are and Basar, Tamer Coalitional game theory for communication networks IEEE signal processing magazine, vol. 26, no. 5, pp. 77-97, 2009.

[8] Karsten, Frank and Slikker, Marco and Van Houtum, Geert-Jan Resource pooling and cost allocation among independent service providers Operations Research, vol. 63, no. 2, pp. 476-488, 2015, INFORMS.

[9] Karsten, Frank and Slikker, Marco and van Houtum, Geert-Jan Analysis of resource pooling games via a new extension of the Erlang loss function Tech. Rep., BETA working paper 344, Eindhoven University of Technology, 2011.

[10] Sundaram, Rangarajan K and others, A first course in optimization theory Cambridge university press, 1996.

[11] http://www.columbia.edu/ ww2040/CallCenterF04/questions1a.pdf

\section{AppendiX A: Characteristic Form Games}

Both partition-form and classic (non-partition) cooperative games can be described in characteristic form [2] using the tuple, $(\mathcal{N}, \nu, \mathcal{H})$, where: a) $\nu$ is called a characteristic function and for any $C \subseteq \mathcal{N}, \nu(C)$ denotes the set of all possible payoff vectors of dimension $n$ that agents in $C$ can jointly achieve; b) $\mathcal{N}$ denotes the set of $n$ agents; and c) $\mathcal{H}$ is the set of all possible payoff vectors of dimension $n$ (such vectors are also referred to as allocation vectors in literature), which are achievable.

In this appendix, we provide the details of how our problem can be recast as a characteristic game.

Let $\mathcal{F}(\mathcal{P})$ be the set of all feasible payoff vectors under partition $\mathcal{P}$, these are the vectors that satisfy the following: the sum of payoffs of all agents in any coalition $S$ is less than or equal to that obtained by $S$ under partition $\mathcal{P}$ at WE, $\lambda_{S}^{\mathcal{P}}$. Thus

$$
\mathcal{F}(\mathcal{P}):=\left\{\mathbf{x}=\left[x_{i}\right]: \sum_{i \in S} x_{i} \leq \lambda_{S}^{\mathcal{P}} \forall S \in \mathcal{P}\right\}
$$

Thus $\mathcal{H}$, the set of all achievable/feasible payoff vectors is,

$$
\mathcal{H}=\cup_{\mathcal{P}} \mathcal{F}(\mathcal{P})
$$

Characteristic function using pessimistic rule: To study the stability aspects, one needs to understand if a certain coalition can 'block' any payoff vector. Blocking by a coalition implies that coalition is working as an independent unit and has an anticipation of the value it can achieve (irrespective of arrangements of others). If the division of this anticipated value among the members of the coalition, under any given allocation rule, renders the members to achieve more than that in the given payoff vector, then the coalition has tendency to oppose the payoff vector.

The characteristic function precisely describes the set of all possible divisions of the anticipated worth of any coalition.

There are many anticipatory rules to define characteristic function for partition form games. The above described rule is the well known pessimistic anticipation rule [4], where the agents in deviating coalition $C$ assume that the outside agents arrange themselves to hurt the agents in $C$ the most.

Towards specifying such a characteristic function, first observe that the minimum utility that coalition $C$ can achieve irrespective of the arrangement of the agents outside this coalition is given by:

$$
\underline{\nu}_{C}:=\min _{\mathbf{x} \in \mathcal{F}(\mathcal{P}): C \in \mathcal{P}} \sum_{i \in C} x_{i} .
$$

With this definition, $\nu(C)$, the set of possible payoff vectors that agents in $C$ can jointly achieve independent of the arrangement of outside agents is given by,

$$
\nu(C)=\left\{\mathrm{x} \in \mathcal{H}: \sum_{i \in C} \mathbf{x}_{i} \leq \underline{\nu}_{C}\right\} .
$$

With these definitions in place, we can now define when a payoff vector is blocked by a coalition.

Blocking: A payoff vector $\mathrm{x} \in \mathcal{H}$ is blocked by a coalition $C$ if there exist a payoff vector $\mathbf{y} \in \nu(C)$ such that

$$
y_{i}>x_{i} \forall i \in C \text {. }
$$

Next, we define $\alpha$-core, which is an extension of the classical definition of core, for transferable utility games (in non-partition form games).

$\alpha$-core: It is the set of all feasible payoff vectors, i.e., $\mathbf{x} \in \mathcal{H}$ such that it is not blocked by any coalition $C$. In other words $\alpha$-core is coalitionally rational: it consists of all feasible payoff vectors such that no coalition of agents can deviate and achieve better. 


\section{APPENDix B: Proof OF THEOREM 1}

Proof of Existence and Uniqueness: Let the size of a partition be denoted by $p$. The first step of this proof is to show the existence and uniqueness of WE for the case when $p=2$. In the next step, using induction we prove the existence for any general $p=m>2$ using the results for $m-1$. In the third step we show the continuity of the WE, to be precise the arrival rates at WE for $m$. The last step attributes to the uniqueness of our solution.

Step 1: Existence and Uniqueness of WE for $p=2$

To obtain WE, the following equation need to be solved:

$$
B_{C_{1}}\left(N_{C_{1}}, a_{C_{1}}\right)=B_{C_{2}}\left(N_{C_{2}}, a_{C_{2}}\right) .
$$

Define a function $f:=B_{C_{1}}\left(N_{C_{1}}, a_{C_{1}}\right)-B_{C_{2}}\left(N_{C_{2}}, a_{C_{2}}\right)$. Then, $f$ is a function of $\lambda_{C_{1}}^{\mathcal{P}} \in[0, \Lambda]$ since $\lambda_{C_{2}}^{\mathcal{P}}=\Lambda-\lambda_{C_{1}}^{\mathcal{P}}$.

- At $\lambda_{C_{1}}^{\mathcal{P}}=0$ we have $B_{C_{1}}\left(N_{C_{1}}, a_{C_{1}}\right)=0$ and $B_{C_{2}}\left(N_{C_{2}}, a_{C_{2}}\right)>0$, thus $f(0)<0$.

- At $\lambda_{C_{1}}^{\mathcal{P}_{1}}=\Lambda$ we have $B_{C_{1}}\left(N_{C_{1}}, a_{C_{1}}\right)>0$ and $B_{C_{2}}\left(N_{C_{2}}, a_{C_{2}}\right)=0$, thus $f(\Lambda)>0$.

Then, $B_{C_{1}}\left(N_{C_{1}}, a_{C_{1}}\right)$ and $B_{C_{2}}\left(N_{C_{2}}, a_{C_{2}}\right)$ are polynomial functions with denominator $>1$ and hence are continuous functions. This implies that $f$ is a continuous function.

Thus, $f$ satisfies the hypothesis of Intermediate Value Theorem (IVT). Using IVT, there exists a value of $\lambda_{C_{1}}^{\mathcal{P}}=\lambda^{*} \in(0, \Lambda)$ such that $f\left(\lambda^{*}\right)=0$. The uniqueness of $\lambda^{*}$ follows since $B_{C_{1}}\left(N_{C_{1}}, a_{C_{1}}\right)$ and $B_{C_{2}}\left(N_{C_{2}}, a_{C_{2}}\right)$ are strict increasing functions of $\lambda_{C_{1}}^{\mathcal{P}}$ and $\lambda_{C_{2}}^{\mathcal{P}}$ respectively.

Step 2: Existence for general $p=m>2$

To prove the existence for any general $m>2$, we assume that a unique WE exists for $p=m-1$, i.e., $\lambda_{C_{1}}^{\mathcal{P}}, \cdots, \lambda_{C_{m-1}}^{\mathcal{P}}$ with corresponding common blocking probability $B^{*}$.

With $m$ units we can initially fix $\lambda_{C_{m}}^{\mathcal{P}}=0$ and obtain WE corresponding to the remaining units, which we have assumed to exist. With increase in $\lambda_{C_{m}}^{\mathcal{P}}, \Lambda-\lambda_{C_{m}}^{\mathcal{P}}$ which is the total share of remaining agents, decreases. From part i) of this theorem, we know that the corresponding WE solution for these agents also decreases. This implies that the common blocking probability for $C_{1}, \cdots, C_{m-1}$ reduces while blocking probability of $C_{m}$ increases. Using similar arguments as above and treating $C_{1}, \cdots, C_{m-1}$ as one while defining function for IVT, one can show that WE exists.

Step 3: Continuity of Optimizers, i.e., WE: Consider the following function for $m$ units in partition $\mathcal{P}$ :

$$
g(\Lambda, \lambda):=\sum_{C_{j} \in \mathcal{P} ; 1<j \leq m}\left(B_{C_{1}}-B_{C_{j}}\right)^{2},
$$

where $\lambda$ is the vector of arrival rates for all $C_{j} \in \mathcal{P}$. Then, we define $g^{*}\left(\Lambda, \lambda^{*}\right)=\min _{\left\{\lambda: \sum_{j} \lambda_{j}=\Lambda\right\}} g(\Lambda, \lambda)$. Observe that the (unique) minimizer $\lambda^{*}$ of the function $g$ is the (unique) WE for our queueing model, and that the function $g$ is jointly continuous. Thus, using Maximum Theorem we have that $g^{*}$ and $\lambda^{*}$ is continuous in $\Lambda$.

Step 4: Uniqueness of WE To prove the uniqueness of the WE, we assume the contradiction. Accordingly, we can have the following cases:

Case 1: There exist multiple WEs with same common blocking probability $B^{*}$

This implies that some of the units in partition are obtaining different arrival rates in the multiple WEs such that they have common $B^{*}$. However, this is not possible since blocking probability is an increasing function of arrival rates. Thus, the unit with higher arrival rate in one of the WEs should have higher blocking probability.

Case 2: There exist multiple WEs with different common blocking probability $B^{*}$ and $\hat{B}^{*}$

Without loss of generality, we can assume that $B^{*}<\hat{B}^{*}$. This implies that the arrival rates to the units with common blocking probability $\hat{B}^{*}$ is more (since blocking probability is an increasing function of arrival rate). However, the total arrival rate is fixed at $\Lambda$ which implies that one of the WE does not satisfy $\sum_{C_{j} \in \mathcal{P}} \lambda_{C_{j}}^{\mathcal{P}}=\Lambda$.

Proof of All units used For contradiction, let us assume that the customers split themselves amongst some strict subset of units of partition $\mathcal{P}$. Then, each unit with zero arrivals have a zero blocking probability while units with non-zero arrivals have some strict positive blocking probability. However, this contradicts the fact that the coalitions having zero arrivals should have a higher blocking probability than others at WE.

Hence at WE, each of the units in partition $\mathcal{P}$ obtain non-zero arrival rates.

Proof of part (i) Let $\lambda_{C_{1}}^{\mathcal{P}}, \cdots, \lambda_{C_{k}}^{\mathcal{P}}$ be the individual arrival rates corresponding to partition $\mathcal{P}$ at WE (satisfies (2)) for the coalitions $C_{1}, \cdots, C_{k}$ respectively with the total arrival rate $\Lambda>0$. Let the corresponding common blocking probability be $B^{*}$. When the total arrival rate is increased to $\Lambda^{\prime}$, the individual arrival rates to the providers at WE are changed to $\lambda_{C_{1}}^{\prime \mathcal{P}}, \cdots, \lambda_{C_{k}}^{\prime \mathcal{P}}$ and the corresponding common blocking probability is changed to $\hat{B}^{*}$. Note that these splits to the individual operating units must satisfy:

$$
\sum_{i=1}^{k} \lambda_{C_{i}}^{\mathcal{P}}=\Lambda \text { for any partition } \mathcal{P}
$$


Next we will show that $\lambda_{C_{j}}^{\prime} \leq \lambda_{C_{j}}^{\mathcal{P}}$ is not possible for any $C_{j} \in \mathcal{P}$. Using equation [17, we know that atleast one of the units have higher individual arrival rates at new WE, i.e,

$$
\lambda_{C_{j}}^{\prime \mathcal{P}}>\lambda_{C_{j}}^{\mathcal{P}} \text { for atleast one } C_{j} \in \mathcal{P} .
$$

This means that the common blocking probability at new WE is increased, i.e., $\hat{B}^{*}>B^{*}$. Now since blocking probability is a strictly increasing function of arrival rates, we have that arrival rate to each coalition is increased at new WE for $\Lambda^{\prime}$, i.e., $\lambda_{C_{j}}^{\mathcal{P}}>\lambda_{C_{j}}^{\mathcal{P}}$ for all $C_{j} \in \mathcal{P}$.

Hence, WE is an increasing function of $\Lambda$.

Proof of part (ii) Let $\lambda_{C_{1}}^{\mathcal{P}}, \cdots, \lambda_{C_{k}}^{\mathcal{P}}$ be the individual arrival rates corresponding to partition $\mathcal{P}$ at WE for the coalitions $C_{1}, \cdots, C_{k}$ respectively. Let the corresponding common blocking probability be $B^{*}$. Observe that the blocking probability of $C_{i}$ and $C_{j}$ units also equals $B^{*}$, and hence the merger $M=C_{i} \cup C_{j} \neq \mathcal{N}$ has strictly smaller blocking probability, i.e., $B_{M}<B^{*}$, if the joint arrival rate was $\lambda_{C_{i}}^{\mathcal{P}}+\lambda_{C_{j}}^{\mathcal{P}}$. From (1) the blocking probability is a strictly decreasing function of arrival rate. Thus the new WE after merger is formed with a (strict) bigger arrival rate to the merger, as again at the new WE the new blocking probabilities of all coalitions $C \in \mathcal{P}^{\prime}$ should be equal by (2).

Proof of part (iii) Consider a system with identical servers. We know that when any number of identical servers combine with their arrival rates, the combined blocking probability reduces. This reduction is more when the number of servers combining are more, i.e.,

$$
B(N, a)>B(L N, L a)>B(M N, M a) .
$$

where $0<L<M$ are constants, $B$ is the blocking probability, $N$ is the number of servers and $a$ is the offered load. Now if we consider that the coalition with $N_{C_{1}}$ and $N_{C_{2}}$ servers gets exactly $N_{C_{1}} / N$ and $N_{C_{2}} / N$ share of total arrival rate $\Lambda$ at WE respectively. Using equation (19), we have that coalition with $N_{C_{1}}$ servers has strictly smaller blocking probability. From (2), the blocking probability of each unit at WE is same. So, the arrival rate to coalition with $N_{C_{1}}$ and $N_{C_{2}}$ servers need to be increased and reduced respectively to achieve the WE.

Hence, coalition with $N_{C_{1}}$ and $N_{C_{2}}$ servers satisfy

$$
\frac{\lambda_{C_{1}}^{\mathcal{P}}}{N_{C_{1}}}>\frac{\Lambda}{N}>\frac{\lambda_{C_{2}}^{\mathcal{P}}}{N_{C_{2}}}
$$

\section{APPENDIX C: REST OF THE PROOFS}

Proof of Theorem 2: Consider a partition $\mathcal{P}=\left\{C_{1}, C_{2}, \cdots, C_{k}\right\}$ with cardinality greater than 2. Let $M$ be the merger coalition containing all coalitions of $\mathcal{P}$ except one, i.e.,

$$
M=\cup_{i=2}^{k} C_{i} \text { and } \mathcal{P}^{\prime}=\left\{C_{1}, M\right\} .
$$

Then from Theorem 1 (ii)

$$
\lambda_{M}^{\mathcal{P}^{\prime}}=\underline{\lambda}_{M}^{\mathcal{P}^{\prime}}>\sum_{C_{i} \in M} \lambda_{C_{i}}^{\mathcal{P}}
$$

which is same as the condition required under RB-IA rule.

Hence, there exists a configuration/payoff vector such that each of the members in $M$ obtain strictly better and thus, such a partition is not stable.

Proof of Theorem 3, i) There can be no merger from $\mathcal{P}_{G}$, and we only need to check if an appropriate split can block the given configuration $\left(\mathcal{P}_{G}, \Phi\right)$ where $\Phi$ is any payoff.

a) We first consider all payoff vectors $\Phi$ that satisfy

$$
\sum_{i=2}^{n} \phi_{i}<\Lambda\left(1-\frac{N_{1}}{N}\right)
$$

Let $S:=\{2,3, \cdots, n\}$ be the coalition made of all agents except agent 1 and we will prove that this coalition (split) will block the configuration of the form stated above. From Theorem 1 (iii) since $\underline{\lambda}_{1}<N_{1} / N \Lambda$, coalition $S$ satisfies the following:

$$
\lambda_{S}^{\mathcal{P}^{\prime}}=\underline{\lambda}_{S}>\Lambda\left(1-\frac{N_{1}}{N}\right), \text { where } \mathcal{P}^{\prime}:=\{S,\{1\}\} .
$$

Hence, there exists a configuration/payoff vector such that each of the members in $S$ obtain strictly better and thus, $\mathcal{P}_{G}$ is not stable for such $\Phi$. 
b) Next, we consider all payoff vectors that satisfy

$$
\sum_{i=2}^{n} \phi_{i} \geq \Lambda\left(1-\frac{N_{1}}{N}\right)
$$

For $\left(\mathcal{P}_{G}, \Phi\right)$ to be stable, the payoff vector should satisfy $\sum_{i \in C} \phi_{i} \geq \underline{\lambda}_{C}=\lambda_{C}^{\mathcal{P}^{\prime}}$ (with $\mathcal{P}^{\prime}:=\{C, \mathcal{N} \backslash C\}$ ), for all $C$ (i.e., negating (5)). From Theorem 1 part iii), any $C$ with more than $N / 2$ servers satisfies equation (5), as $\lambda^{\mathcal{P}_{G}}=\Lambda$. We use a subset of such coalitions to complete the proof. Since $N_{1}$ is the agent with maximum number of servers, $S_{k}:=S \backslash\{k\} \cup\{1\}$ has $N_{S_{k}}>N / 2$ for any $k \geq 2$, do not satisfies equation (5) by Theorem 1 part (iii) and will block the given payoff vector $\Phi$ if the following equations are not satisfied simultaneously:

$$
\begin{aligned}
\phi_{1}+\phi_{2}+\phi_{3} \cdots+\phi_{n-1} & >\frac{N_{1}+\sum_{i=2}^{n-1} N_{i}}{N} \Lambda, \\
\phi_{1}+\phi_{2}+\phi_{3} \cdots+\phi_{n-2}+\phi_{n} & >\frac{N_{1}+\sum_{i=2}^{n-2} N_{i}+N_{n}}{N} \Lambda, \\
\vdots & > \\
\phi_{1}+\phi_{3}+\phi_{4} \cdots+\phi_{n} & >\frac{N_{1}+\sum_{i=3}^{n} N_{i}}{N} \Lambda .
\end{aligned}
$$

Adding these $(n-1)$ equations, we obtain the following:

$$
\begin{aligned}
(n-1) \phi_{1}+(n-2) \sum_{i=2}^{n} \phi_{i}> & \frac{(n-1) N_{1}+(n-2) \sum_{i=2}^{n} N_{i}}{N} \Lambda, \\
\phi_{1}+(n-2) \Lambda> & \left(\frac{N_{1}+(n-2) N}{N}\right) \Lambda, \\
& \text { since } \sum_{i=1}^{n} \phi_{i}=\Lambda, \sum_{i=1}^{n} N_{i}=N .
\end{aligned}
$$

Thus for payoff vector $\Phi$ to be not blocked we require that, $\phi_{1}>\frac{N_{1}}{N} \Lambda$ and thus, $\sum_{i=2}^{n} \phi_{i}<\left(1-\frac{N_{1}}{N}\right) \Lambda$ which is not possible since we are considering payoff vectors that satisfy [20].

Thus, $\left(\mathcal{P}_{G}, \Phi\right)$ is not a stable configuration with any payoff vector $\Phi$.

ii) When $N_{1}>\sum_{i \in \mathcal{N} ; i \neq 1} N_{i}$

In such a case, all coalitions that satisfy equation (5) must include agent 1 . Thus, $\Phi$ should satisfy:

$$
\phi_{1}>\max _{C} \underline{\lambda}_{C} \text { for all } C \subset \mathcal{N} \text { satisfying [5] and containing agent } 1 .
$$

Since no other coalition satisfy equation (5), any payoff vector that satisfies the above equation ensures configuration $\left(\mathcal{P}_{G}, \Phi\right)$ to be stable.

Proof of Theorem 4: Any 2-partition $\mathcal{P}=\left\{C_{1}, C_{2}\right\}$ cannot be blocked by mergers since merger lead to $\mathcal{P}_{G}$ and (7) is not satisfied. Next we look at splits. Say $C_{1} \in \mathbb{C}^{*}$. Then it follows from the definition of $\mathbb{C}^{*}$ that there exists no coalition $C \subset C_{1}$ such that it satisfies [5]. For any split of $C_{2}$ into $S$ and $C_{2} \backslash S$, from Theorem 1 (ii) we know that

$$
\lambda_{C_{2}}^{\mathcal{P}}>\lambda_{S}^{\mathcal{P}^{\prime}}+\lambda_{C_{2} \backslash S}^{\mathcal{P}^{\prime}}
$$

Thus, there exists a payoff vector $\Phi$ that allocates strictly better to each player in $C_{2}$ which implies that such a split is not feasible. Hence, any partition with one of the coalitions belonging to $\mathbb{C}^{*}$ is a stable partition under RB-IA rule.

Next, when each coalition has $N / 2$ servers, the per-server share of each coalition equals $\Lambda / N$ at WE. However, if any subset of players (from any of the coalitions) deviate then the per-server share obtained by this coalition is strictly smaller than $\Lambda / N$ (from Theorem 1 (iii)) and hence such a partition is stable.

Proof of Theorem 5: i) Consider any configuration $\left(\mathcal{P}_{G}, \Phi\right\}$ with GC. The proof of this part can be split into two cases: Case 1: When $N_{1} \leq \sum_{i \in S ; i \neq 1} N_{i}$ for some $S \subset \mathcal{N}$

Under RB-PA rule for the configuration to be stable, we need to ensure that the following system of equations are satisfied simultaneously.

$$
\sum_{i \in C} \phi_{i} \geq \underline{\lambda}_{C} \text { for all } C \subset \mathcal{N} \text { and, } \sum_{i \in \mathcal{N}} \phi_{i}=\Lambda .
$$

However, a subset of these equations itself admit no feasible solution (as proved in Theorem 3). Thus, such a system of equations does not have a solution and hence $\left(\mathcal{P}_{G}, \Phi\right\}$ is unstable for any payoff vector $\Phi$.

Case 2: When $N_{1}>\sum_{i \in \mathcal{N} ; i \neq 1} N_{i}$ 
Once again we need to satisfy (21) to prove that $\left(\mathcal{P}_{G}, \Phi\right)$ is stable. In particular those equations will also have to be satisfied for subsets $S$ such that $|S|=n-1$, and $1 \in S$. If there exists a payoff vector $\Phi$ that satisfies all such conditions, consider one such $S$ and say $j \notin S$. Then from 21):

$$
\phi_{j}=\Lambda-\sum_{i \in S} \phi_{i} \leq \Lambda-\underline{\lambda}_{S}=\underline{\lambda}_{\{j\}},
$$

If $\phi_{j}<\underline{\lambda}_{\{j\}}$ for some $j$ then configuration $\left(\mathcal{P}_{G}, \Phi\right)$ is blocked by $\{j\}$ under RB-PA rule. Otherwise if $\phi_{j}=\underline{\lambda}_{\{j\}}$ for all $j \in \mathcal{N}$ then $\sum_{i \in \mathcal{N}} \phi_{i}=\sum_{i \in \mathcal{N}} \underline{\lambda}_{\{j\}}<\Lambda$ and thus (21) is not satisfied. Hence $\left(\mathcal{P}_{G}, \Phi\right)$ is unstable for any payoff $\Phi$.

Proof of part ii): Since the condition required for a merger to be successful under RB-PA rule is same as under RB-IA rule, the result follows from Theorem 2.

Proof of parts iii) and iv): When the payoff vector is given by equation (9), the RB-PA and RB-IA rules are equivalent to each other. Thus, the result follows from Theorem 4.

Proof of part v): The first part of this result follows from parts iii) and iv) of this theorem.

Moreover because of the continuity of $\Phi$, we have the next result.

Proof of Theorem 77: The proof of Theorem 7 is available in main text in this report on page 8.

Proof of Lemma 2; i) The equation (10) can be re-written as: (by replacing $\lambda_{1} / k$ with $\psi$ and $\lambda_{2}=\Lambda-k \psi$ )

$$
\psi=\frac{\Lambda-k \psi}{N-k}\left(\frac{1+\frac{1}{\psi}-\frac{1}{k \psi}}{1+\frac{N-k}{\Lambda-k \psi}-\frac{1}{\Lambda-k \psi}}\right) .
$$

Simplifying it further, we have

$$
\psi\left(1+\frac{N-k-1}{\Lambda-k \psi}\right)=\frac{\Lambda-k \psi}{N-k}\left(1+\frac{1}{\psi}-\frac{1}{k \psi}\right) .
$$

Relaxing $k$ to a real number and then differentiating the above equation with respect to $k$, we have

$$
\begin{aligned}
\frac{\partial}{d k}\left[\psi\left(1+\frac{N-k-1}{\Lambda-k \psi}\right)\right]= & \frac{\partial \psi}{\partial k}\left(1+\frac{N-k-1}{\Lambda-k \psi}\right)+\psi\left(\frac{\partial}{d k}\left\{\frac{N-k-1}{\Lambda-k \psi}\right\}\right) \\
= & \frac{\partial \psi}{\partial k}\left(1+\frac{N-k-1}{\Lambda-k \psi}\right)+\psi\left(\frac{-(\Lambda-k \psi)+(N-k-1)\left(\psi+k \frac{\partial \psi}{\partial k}\right)}{(\Lambda-k \psi)^{2}}\right), \\
= & \frac{\partial \psi}{\partial k}\left(1+\frac{N-k-1}{\Lambda-k \psi}+\frac{k \psi(N-k-1)}{(\Lambda-k \psi)^{2}}\right)+\psi\left(\frac{-(\Lambda-k \psi+(N-k-1) \psi}{(\Lambda-k \psi)^{2}}\right) \cdot(23) \\
& \frac{\partial}{d k}\left[\frac{\Lambda-k \psi}{N-k}\left(1+\frac{1}{\psi}-\frac{1}{k \psi}\right)\right] \\
= & \left(1+\frac{1}{\psi}-\frac{1}{k \psi}\right) \frac{\partial}{d k}\left\{\frac{\Lambda-k \psi}{N-k}\right\}+\left(\frac{\Lambda-k \psi}{N-k}\right) \frac{\partial}{d k}\left\{\left(1+\frac{1}{\psi}-\frac{1}{k \psi}\right),\right\} \\
= & \left(1+\frac{1}{\psi}-\frac{1}{k \psi}\right)\left(\frac{-(N-k)\left(\psi+k \frac{\partial \psi}{\partial k}\right)+(\Lambda-k \psi)}{(N-k)^{2}}\right)+\left(\frac{\Lambda-k \psi}{N-k}\right)\left(-\frac{1}{\psi^{2}} \frac{\partial \psi}{\partial k}+\frac{1}{k^{2} \psi^{2}}\left(\psi+k \frac{\partial \psi}{\partial k}\right)\right) \\
= & \frac{\partial \psi}{\partial k}\left[-\frac{k}{N-k}\left(1+\frac{1}{\psi}-\frac{1}{k \psi}\right)-\left(\frac{1}{\psi^{2}}-\frac{1}{k \psi^{2}}\right)\left(\frac{\Lambda-k \psi}{N-k}\right)\right]+\left[-\frac{\psi}{N-k}\left(1+\frac{1}{\psi}-\frac{1}{k \psi}\right)+\frac{(\Lambda-k \psi)}{(N-k)^{2}}\left(1+\frac{1}{\psi}-\frac{1}{k \psi}\right)\right. \\
& \left.+\frac{1}{k^{2} \psi}\left(\frac{\Lambda-k \psi}{N-k}\right)\right] \cdot
\end{aligned}
$$

Combining the derivatives of LHS and RHS, we have

$$
\begin{array}{r}
\frac{\partial \psi}{\partial k}\left[1+\frac{N-k-1}{\Lambda-k \psi}+\frac{k \psi(N-k-1)}{(\Lambda-k \psi)^{2}}+\frac{k}{N-k}\left(1+\frac{1}{\psi}-\frac{1}{k \psi}\right)+\left(\frac{1}{\psi^{2}}-\frac{1}{k \psi^{2}}\right)\left(\frac{\Lambda-k \psi}{N-k}\right)\right]= \\
{\left[-\frac{\psi}{N-k}\left(1+\frac{1}{\psi}-\frac{1}{k \psi}\right)+\frac{(\Lambda-k \psi)}{(N-k)^{2}}\left(1+\frac{1}{\psi}-\frac{1}{k \psi}\right)+\frac{1}{k^{2} \psi}\left(\frac{\Lambda-k \psi}{N-k}\right)-\psi\left(\frac{-(\Lambda-k \psi)+(N-k-1) \psi}{(\Lambda-k \psi)^{2}}\right)\right] .}
\end{array}
$$


Hence,

$$
\frac{\partial \psi}{\partial k}=\frac{-\frac{\psi}{N-k}\left(1+\frac{1}{\psi}-\frac{1}{k \psi}\right)+\frac{(\Lambda-k \psi)}{(N-k)^{2}}\left(1+\frac{1}{\psi}-\frac{1}{k \psi}\right)+\frac{1}{k^{2} \psi}\left(\frac{\Lambda-k \psi}{N-k}\right)-\psi\left(\frac{-(\Lambda-k \psi)+(N-k-1) \psi}{(\Lambda-k \psi)^{2}}\right)}{1+\frac{N-k-1}{\Lambda-k \psi}+\frac{k \psi(N-k-1)}{(\Lambda-k \psi)^{2}}+\frac{k}{N-k}\left(1+\frac{1}{\psi}-\frac{1}{k \psi}\right)+\left(\frac{1}{\psi^{2}}-\frac{1}{k \psi^{2}}\right)\left(\frac{\Lambda-k \psi}{N-k}\right)}
$$

The denominator is always strictly positive. When $\Lambda \rightarrow \infty$, denominator tends to $1+\frac{k}{N-k}>0$. The numerator can be re-written as:

$$
\begin{aligned}
& -\frac{\psi}{N-k}\left(1+\frac{1}{\psi}-\frac{1}{k \psi}\right)+\frac{(\Lambda-k \psi)}{(N-k)^{2}}\left(1+\frac{1}{\psi}-\frac{1}{k \psi}\right)+\frac{1}{k^{2} \psi}\left(\frac{\Lambda-k \psi}{N-k}\right)-\psi\left(\frac{-(\Lambda-k \psi)+(N-k-1) \psi}{(\Lambda-k \psi)^{2}}\right) \\
= & \frac{1}{N-k}\left(1+\frac{1}{\psi}-\frac{1}{k \psi}\right)\left[-\psi+\frac{\Lambda-k \psi}{N-k}\right]+\frac{1}{k^{2} \psi}\left(\frac{\Lambda-k \psi}{N-k}\right)+\psi\left(\frac{\Lambda-(N-1) \psi}{(\Lambda-k \psi)^{2}}\right) \\
= & \frac{1}{(N-k)^{2}}\left(1+\frac{1}{\psi}-\frac{1}{k \psi}\right)(\Lambda-N \psi)+\frac{1}{k^{2} \psi}\left(\frac{\Lambda-k \psi}{N-k}\right)+\psi\left(\frac{\Lambda-(N-1) \psi}{(\Lambda-k \psi)^{2}}\right)
\end{aligned}
$$

Using equation (11) we have

$$
\begin{gathered}
\frac{1}{(N-k)^{2}}\left(1+\frac{1}{\psi}-\frac{1}{k \psi}\right)(\Lambda-N \psi)=\frac{1}{(N-k)^{2}}\left(1+\frac{1}{\psi}-\frac{1}{k \psi}\right) N \Lambda \frac{\left(\frac{\Lambda}{N}-\psi\right)}{\Lambda} \rightarrow 0 . \\
\frac{1}{k^{2} \psi}\left(\frac{\Lambda-k \psi}{N-k}\right)=\frac{1}{k^{2} \psi}\left(\frac{\Lambda-N \psi+N \psi-k \psi}{N-k}\right)=\frac{1}{k^{2} \psi}\left(\frac{\Lambda-N \psi}{N-k}\right)+\frac{1}{k^{2} \psi}\left(\frac{N \psi-k \psi}{N-k}\right) \rightarrow \frac{1}{k^{2}}
\end{gathered}
$$

since

$$
\frac{1}{k^{2} \psi}\left(\frac{\Lambda-N \psi}{N-k}\right)=\frac{\Lambda}{k^{2} \psi}\left(\frac{\Lambda-N \psi}{(N-k) \Lambda}\right)=\frac{N \Lambda}{k^{2} \psi}\left(\frac{\frac{\Lambda}{N}-\psi}{(N-k) \Lambda}\right) \rightarrow 0 .
$$

From the similar arguments as above, we can observe that

$$
\psi\left(\frac{\Lambda-(N-1) \psi}{(\Lambda-k \psi)^{2}}\right)=\psi\left(\frac{\Lambda-N \psi}{(\Lambda-k \psi)^{2}}\right)+\psi\left(\frac{\psi}{(\Lambda-k \psi)^{2}}\right) \rightarrow\left(\frac{\psi}{\Lambda-k \psi}\right)^{2}>0
$$

Hence, we have

$$
\frac{\partial \psi}{\partial k}>0
$$

Thus, there exists a $\Lambda$ such that for all $\Lambda \geq \bar{\Lambda}, \psi$ is increasing with $k$.

ii) Since, $\psi$ is increasing with $k$ for any $\Lambda \geq \bar{\Lambda}$, the unique maximiser of $(8)$ is obtained at maximum possible of $k$ which is given by $\sum_{i=1}^{N-1} N_{i}$ (since $N_{i}$ are arranged in decreasing order).

iii) When any coalition in $\mathcal{P}$, say $C_{1}$ with $N / 2 \leq N_{C_{1}} \leq k^{*}$ splits into $S$ and $C_{1} \backslash S$ to form $\mathcal{P}^{\prime}$, then using similar arguments as in Theorem 1 (ii)

$$
\lambda_{C_{1}}^{\mathcal{P}}>\lambda_{S}^{\mathcal{P}^{\prime}}+\lambda_{C_{1} \backslash S}^{\mathcal{P}^{\prime}}
$$

Thus, we can have a payoff vector $\Phi$ such that each player is strictly better in $C_{1}$. Hence, all such 2-partitions are stable under second order approximation.

Proof of Lemma 3; i) Recall the WE is obtained by equating the reciprocal of the blocking probabilities. From (1), at low traffic this can approximately be achieved by (solving for $\lambda_{1}$ which is a zero of the following):

$$
\frac{k !}{\lambda_{1}^{k}}=\frac{(N-k) !}{\lambda_{2}^{N-k}} \text { or } \lambda_{2}=\left\{\frac{(N-k) !}{k !}\right\}^{1 /(N-k)} \lambda_{1}^{k /(N-k)} .
$$

Using $\lambda_{2}=\Lambda-\lambda_{1}$ we get

$$
\frac{\lambda_{1}}{\Lambda}=\frac{1}{1+\left\{\frac{(N-k) !}{k !}\right\}^{1 /(N-k)} \lambda_{1}^{\frac{k}{N-k}-1}} .
$$

Since $k>N-k$ we have $\frac{k}{N-k}>1$. As $\Lambda \rightarrow 0,\left\{\frac{(N-k) !}{k !}\right\}^{1 /(N-k)} \lambda_{1}^{\frac{k}{N-k}-1} \rightarrow 0$ and thus,

$$
\frac{\lambda_{1}}{\Lambda} \rightarrow 1 \text { as } \Lambda \rightarrow 0
$$


Suppose the coalition with higher number of servers has $\left\lfloor\frac{N}{2}\right\rfloor+m$ servers. From equation 27] we have

$$
\frac{\lambda_{1}}{\Lambda\left(\left\lfloor\frac{N}{2}\right\rfloor+m\right)} \rightarrow \frac{1}{\left(\left\lfloor\frac{N}{2}\right\rfloor+m\right)} \text { as } \Lambda \rightarrow 0
$$

Thus the arrival rate allotted to each server in this coalition as a fraction of total arrival rate lies in the interval

$$
\frac{1}{\left(\left\lfloor\frac{N}{2}\right\rfloor+m\right)}-\epsilon<\frac{\lambda_{1}}{\Lambda k}<\frac{1}{\left(\left\lfloor\frac{N}{2}\right\rfloor+m\right)}+\epsilon .
$$

Choose an $\epsilon>0$ such that the lower bound with minimum possible value of $m$, i.e., $\tilde{m}$ is greater than the upper bound for all $m>\tilde{m}$. Thus, we want to show:

$$
\frac{1}{\left(\left\lfloor\frac{N}{2}\right\rfloor+\tilde{m}\right)}-\epsilon>\frac{1}{\left(\left\lfloor\frac{N}{2}\right\rfloor+m\right)}+\epsilon \quad \text { for all } m>\tilde{m} .
$$

It is sufficient to show that the upper bound for the least value of $m$ greater than $\tilde{m}$, i.e. $\hat{m}$ is smaller for some $\epsilon$, i.e.,

$$
\begin{aligned}
\frac{1}{\left(\left\lfloor\frac{N}{2}\right\rfloor+\tilde{m}\right)}-\epsilon & >\frac{1}{\left(\left\lfloor\frac{N}{2}\right\rfloor+\hat{m}\right)}+\epsilon, \\
\frac{\hat{m}-\tilde{m}}{\left(\left\lfloor\frac{N}{2}\right\rfloor+\tilde{m}\right)\left(\left\lfloor\frac{N}{2}\right\rfloor+\hat{m}\right)} & >2 \epsilon, \\
\frac{\hat{m}-\tilde{m}}{2\left(\left\lfloor\frac{N}{2}\right\rfloor+\tilde{m}\right)\left(\left\lfloor\frac{N}{2}\right\rfloor+\hat{m}\right)} & >\epsilon>0 .
\end{aligned}
$$

ii) The partitions with one of the coalitions having servers between $N / 2 \leq N_{C} \leq k^{*}$ is stable can be proved using similar arguments as in Lemma 2 2iii). For partition with $N_{C}>k^{*}$, players with $k^{*}$ servers can deviate together to obtain higher individual shares. This is because the players still obtain approximately same coalitional share but the number of players to share are less. 